\title{
Expansion and diversification of the SET domain gene family following whole-genome duplications in Populus trichocarpa
}

\author{
Li Lei ${ }^{1,3}$, Shi-Liang Zhou', Hong Ma ${ }^{2^{*}}$ and Liang-Sheng Zhang ${ }^{2^{*}}$
}

\begin{abstract}
Background: Histone lysine methylation modifies chromatin structure and regulates eukaryotic gene transcription and a variety of developmental and physiological processes. SET domain proteins are lysine methyltransferases containing the evolutionarily-conserved SET domain, which is known to be the catalytic domain.

Results: We identified 59 SET genes in the Populus genome. Phylogenetic analyses of 106 SET genes from Populus and Arabidopsis supported the clustering of SET genes into six distinct subfamilies and identified 19 duplicated gene pairs in Populus. The chromosome locations of these gene pairs and the distribution of synonymous substitution rates showed that the expansion of the SET gene family might be caused by large-scale duplications in Populus. Comparison of gene structures and domain architectures of each duplicate pair indicated that divergence took place at the $3^{\prime}$ - and 5'-terminal transcribed regions and at the $\mathrm{N}$ - and C-termini of the predicted proteins, respectively. Expression profile analysis of Populus SET genes suggested that most Populus SET genes were expressed widely, many with the highest expression in young leaves. In particular, the expression profiles of 12 of the 19 duplicated gene pairs fell into two types of expression patterns.

Conclusions: The 19 duplicated SET genes could have originated from whole genome duplication events. The differences in SET gene structure, domain architecture, and expression profiles in various tissues of Populus suggest that members of the SET gene family have a variety of developmental and physiological functions. Our study provides clues about the evolution of epigenetic regulation of chromatin structure and gene expression.
\end{abstract}

\section{Background}

Gene expression is regulated by many factors acting in concert with the status of the chromatin environment, particularly histone methylation [1]. The SET-domain-containing protein family is a major player in histone methylation. These proteins are responsible for the methylation of lysine (K) residues in various histones, specifically $\mathrm{K} 4, \mathrm{~K} 9, \mathrm{~K} 27$, and $\mathrm{K} 36$ in histone $\mathrm{H} 3$ and $\mathrm{K} 20$ in histone H4 [2]; H3K79 is an exception [3]. All members of this family share a highly conserved SET domain [2], named for three Drosophila melanogaster proteins: Suppressor of variegation 3-9 (Su(var)3-9) (Suv), Enhancer of Zeste (E(z)) and Trithorax (Trx). This domain has approximately 130 amino acids and

\footnotetext{
* Correspondence: hongma@fudan.edu.cn; zls@fudan.edu.cn

${ }^{2}$ State Key Laboratory of Genetic Engineering, Institute of Plants Biology, Center for Evolutionary Biology, School of Life Sciences, Fudan University, Shanghai 200433, China

Full list of author information is available at the end of the article
}

has been found in all eukaryotic organisms studied so far [2]. Proteins containing the SET domain can also be found in viruses as well as both domains of prokaryotes $[4,5]$.

Recent studies have revealed that the SET domain (here after referred as $S E T$ ) genes are important for regulating growth and reproduction processes, such as control of flowering time and embryogenesis in plants [6]. Genome sequencing has uncovered many genes encoding SETdomain proteins; in particular, Arabidopsis SET genes are the best annotated and characterized. For example, the Arabidopsis CURLY LEAF (CLF) gene is required for stable repression of the floral homeotic gene AGAMOUS in leaves and stems [7]. ARABIDOPSIS TRITHORAX 1 (ATX1) functions as an activator of homeotic genes, like Trithorax in animal systems [8]. ARABIDOPSIS TRITHORAX-RELATED PROTEIN 7 (ATXR7) is an H3K4 methylase required for proper expression of the

\section{Biomed Central}


Flowering Locus $C$ ( $F L C)$ gene [9]. The Arabidopsis ASH1 HOMOLOG 2 (ASHH2) protein has been suggested to methylate H3K4 and/or H3K36, similar to Drosophila ASH1 and yeast SET2, a H3K36 histone methyltransferase (HMT) [10]. Other SET genes are associated with embryogenesis, including $M E D E A$ (MEA); a maternally inherited loss-of-function mea allele results in embryo abortion and prolonged endosperm production [11]. Recently, ATXR3 is crucial for both sporophyte and gametophyte development and encodes the major enzyme responsible for trimethylation of H3K4 [12,13].

In plants, at least $47,33,31$, and $43 S E T$ genes have been identified in Arabidopsis, grape, maize and rice, respectively [14-16]. In both Arabidopsis and rice it has been determined that many SET genes are located in large blocks of related regions derived from whole genome duplication events, indicating that whole genome duplication could be an important contributor to the duplication of $S E T$ genes [15]. In addition, different classifications of $S E T$ genes were used in different plants. Initially, 37 putative Arabidopsis SET domain proteins were classified into four distinct classes: (I) enhancer of zeste $[\mathrm{E}(\mathrm{z})]$ homologs; (II) trithorax (Trx) homologs and related proteins; (III) Ash1 homologs and related proteins; and (IV) suppressor of variegation [Su(var)] homologs and related proteins [17]. In another study, 32 Arabidopsis and 22 maize SET genes were classified into five classes according to phylogenetic relationships and domain organization [18]. More recently, two additional classes (VI and VII) were recognized for SET genes in Arabidopsis, grape, maize, and rice [14-16]. Interestingly, in Arabidopsis several genes in class III, like ATXR3, were shown to be crucial for both sporophyte and gametophyte development [12,13]. Moreover, Arabidopsis has ten $\mathrm{Su}(\mathrm{var})$ homologue (SUVH) genes belonging to Class V, including several that control heterochromatic domains. Loss of function of these genes suppresses gene silencing, whereas overexpression enhances silencing, causing ectopic heterochromatization and significant growth defects in Arabidopsis [19]. Therefore, $S E T$ genes in different subfamilies could have diverse functions.

Previous studies of SET genes have focused on annotation and Arabidopsis functional characterizations [7-13,19]; in addition evolutionary analyses have been limited to herbaceous plants [14-18]. Trees are distinct from herbaceous species in many ways: they have a selfsupporting structure, the secondary growth or wood, and a much longer lifespan $[20,21]$. The regulatory networks and molecular mechanisms that underlie these unique properties cannot be investigated through the examination of nontree species. Therefore, it is worthwhile to study $S E T$ genes in trees, thereby improving our understanding in their functions and the evolution of SET genes. The recently completed genome sequence of the model tree, Populus trichocarpa (hereafter called Рориlus) [22], provides a great opportunity to investigate these issues.

Molecular evidence suggests that Arabidopsis and Populus shared their last common ancestor as much as 100 to 120 million years ago [22]. Since then, Arabidopsis and Populus have evolved different life histories, including herbaceous versus arboreal development, annual versus perennial habit, and self-pollination versus cross-pollination strategies $[20,21]$. In addition, since they diverged from each other, Populus has experienced whole genome duplication once, whereas Arabidopsis has twice [22,23]. In plants, evolutionary diversity has been hypothesized to be modulated directly or indirectly by epigenetic regulations [24]. Therefore, the SET gene family, among the most important epigenetic regulators, could be postulated to contribute substantially to the evolutionary innovations in plant diversity.

We conducted a comparative analysis of SET genes from Arabidopsis and Populus to address the key question: how have SET genes evolved in Populus after the divergence of Arabidopsis and Populus. In particular, how did the SET gene family expand and diversify in Populus? In this study, we performed comprehensive analyses of SET genes from Populus, including phylogeny, gene structure, domain architecture, gene duplication and diversification, and expression profiling analyses. Our results provide insight into the function of Populus $S E T$ genes and provide a basis for understanding how gene functions, particularly functions involved in the development of trees, have evolved.

\section{Results}

\section{Identification of SET genes in Populus}

We obtained the Populus whole-genome shotgun trace data from JGI and identified all predicted proteins containing SET domains. We named Populus SET genes based on the previously reported Arabidopsis SET gene names [17] and phylogenetic relationships here (Table 1). The Arabidopsis SET genes follow the standard gene symbol conventions with all capital letters; for genes from Populus, the first letter was capitalized but others were in lower case, using the same name as the closest Arabidopsis homolog. If the name of the closest Arabidopsis homolog is already used for a Populus gene, then the name of the next closest homolog is used; if two or more Populus genes are equally close to a single Arabidopsis gene, then the same name is used followed by the letters "a", "b", etc. A total of 59 Populus SET genes were identified and compared to SET genes in Arabidopsis (47) and rice (43) [15]. There were a total of 45,555 protein-coding genes in Populus, 27,417 in Arabidopsis and 56,662 in rice. This suggested that the numbers of SET 
Table 1 Members of the SET gene family in Populus

\begin{tabular}{|c|c|c|c|c|c|}
\hline Gene name & Protein length & subfamily* & ESTs & Gene position & Gene Model \\
\hline PoSuvh2 & 453 & Suv & - & LG_VIII(11654980-11656338) & gw1.VIII.652.1 \\
\hline PoSuvh9 & 519 & Suv & 6 & LG_X(5498008-5499564) & gw1.X.4649.1 \\
\hline PoSuvh5 & 513 & Suv & - & LG_III(9558215-9561181) & gw1.118.279.1 \\
\hline PoSuvh4a & 486 & Suv & - & LG_III(14574409-14583315) & gw1..II.353.1 \\
\hline PoSuvh4b & 525 & Suv & 8 & LG_II(22271452-22288911) & gw1.1I.373.1 \\
\hline PoSurh4c & 509 & Suv & - & LG_XIV(7067483-7077824) & gw1.XIV.3033.1 \\
\hline PoSuvh3 & 496 & Suv & - & LG_VI(1774010-1775496) & gw1.VI.98.1 \\
\hline PoSuvh10 & 496 & Suv & 7 & LG_XVI(1303523-1305010) & gw1.XVI.1034.1 \\
\hline PoSuvh1 & 653 & Suv & 2 & LG_III(17984592-17986613) & fgenesh4_pg.C_LG_III001771 \\
\hline PoSuvh7 & 512 & Suv & 7 & LG_I(1436489-1438607) & estExt_Genewise1_v1.C_LG_16844 \\
\hline PoSuvr5a & 1428 & Suv & - & LG_V(4008809-4012099) & gw1.V.3747.1 \\
\hline PoSuvr5b & 1517 & Suv & 5 & LG_VII(10134577-10137249) & estExt_Genewise1_v1.C_LG_VII3275 \\
\hline PoSuvr1 & 296 & Suv & - & LG_IV(16108605-16109961) & gw1.IV.1292.1 \\
\hline PoSuvr2 & 414 & Suv & - & LG_IX(1847452-1849907) & gw1.IX.1069.1 \\
\hline PoSuvr4 & 401 & Suv & 3 & LG_XIII(3470303-3478528) & gw1.XIII.1724.1 \\
\hline PoSuvr4a & 714 & Suv & 2 & LG_XIV(12538712-12542929) & gw1.XIV.76.1 \\
\hline PoSuvr4b & 464 & Suv & 4 & LG_II(23795948-23799204) & gw1.II.215.1 \\
\hline PoSuvr3 & 340 & Suv & 5 & LG_XIII(4848844-4850695) & gw1.XIII.2167.1 \\
\hline PoAtxr6a & 319 & Atxr5 & - & LG_XII(2169730-2172278) & gw1.XII.888.1 \\
\hline PoAtxr6b & 325 & Atxr5 & - & LG_XV(668228-670843) & gw1.XV.292.1 \\
\hline PoAtxr5a & 304 & Atxr5 & 9 & LG_VII(2505750-2509040) & fgenesh4_pm.C_LG_VII000124 \\
\hline PoAtxr5b & 333 & Atxr5 & 1 & LG_V(3899727-3900751) & gw1.70.74.1 \\
\hline PoAshh3 & 351 & Ash & 8 & LG_XVII(191202-195604) & eugene3.01700004 \\
\hline PoAshh4 & 281 & Ash & 7 & LG_VII(13836-18843) & eugene3.00070002 \\
\hline PoAshh1 & 495 & Ash & 8 & LG_V(13318562-13324389) & fgenesh4_pm.C_LG_V000437 \\
\hline PoAshr3 & 402 & Ash & - & LG_XVIII(10390889-10400394) & gw1.XVIII.3091.1 \\
\hline PoAshh2a & 605 & Ash & 1 & LG_I(5451879-5460030) & eugene3.00020732 \\
\hline PoAshh2b & 594 & Ash & - & LG_V(1177943-12003046) & gw1.V.27.1 \\
\hline PoAtxr3a & 2350 & Atxr3 & 7 & LG_VII(1678369-1689434) & fgenesh4_pg.C_LG_VII000266 \\
\hline PoAtxr3b & 2476 & Atxr3 & 28 & LG_XVII(5241549-5229352) & eugene 3.00640010 \\
\hline Poswna & 852 & $E(z)$ & 10 & LG_II(16109075-16116062) & gw1.II.890.1 \\
\hline PoSwnb & 812 & $E(z)$ & 1 & LG_XIV(5213392-5220923) & estExt_Genewise1_v1.C_LG_XIV2284 \\
\hline PoClfa & 917 & $E(z)$ & 5 & LG_V(5117508-5125370) & estExt_fgenesh4_pg.C_LG_V0535 \\
\hline PoClfb & 892 & $E(z)$ & 1 & LG_VII(8674891-8681980) & estExt_Genewise1_v1.C_LG_VII2923 \\
\hline PoAtxr7a & 1390 & $\operatorname{Trx}$ & 2 & LG_II(69963-76718) & fgenesh4_pg.C_LG_II000009 \\
\hline PoAtxr7b & 1149 & $\operatorname{Trx}$ & 3 & LG_V(25489363-25495946) & POPTR_0005s28130 \\
\hline PoAtx6 & 712 & Trx & - & LG_XVIII(1929427-1923848) & POPTR_0018s02170 \\
\hline PoAtx1 & 1014 & Trx & 6 & LG_II(20140580-20153598) & fgenesh4_pg.C_LG_II002136 \\
\hline$\overline{\text { PoAtx2 }}$ & 1050 & Trx & 5 & LG_XIV(7247301-7259403) & gw1.XIV.3109.1 \\
\hline PoAtx3a & 667 & Trx & 2 & LG_II(13105976-13113638) & fgenesh4_pg.C_LG_II001571 \\
\hline PoAtx3b & 908 & $\operatorname{Trx}$ & 6 & LG_XIV(3468877-3477546) & gw1.XIV.1697.1 \\
\hline PoAtx5 & 1070 & $\operatorname{Trx}$ & 3 & LG_XV(992498-1001204) & gw1.XV.426.1 \\
\hline PoAtx4 & 1078 & $\operatorname{Trx}$ & 4 & LG_XII(1824820-1833670) & gw1.XII.196.1 \\
\hline PoAshr2 & 326 & SMYD & 3 & LG_I(5175894-5176871) & gw1.1.8071.1 \\
\hline PoAtxr4 & 283 & SMYD & 7 & LG_VI(5075142-10640133) & gw1.VI.14.1 \\
\hline PoAtxr1 & 542 & SMYD & - & LG_VIII(5563751-5563753) & fgenesh4_pg.C_LG_VIII000780 \\
\hline PoAtxr2 & 398 & SMYD & 9 & LG_XVII(5018556-5014447) & estExt_fgenesh4_pm.C_640013 \\
\hline
\end{tabular}


Table 1 Members of the SET gene family in Populus (Continued)

\begin{tabular}{llllll}
\hline PoAshr1 & 458 & SMYD & 4 & LG_V(11020007-11025452) & eugene3.00050808 \\
\hline PoSetd8 & 167 & SETD & 4 & LG_III(13995189-13996722) & gw1.III.385.1 \\
\hline PoSetd3 & 362 & SETD & 1 & LG_VIII(8841653-8843777) & fgenesh4_pg.C_LG_VIII001195 \\
\hline PoSetd4 & 513 & SETD & 2 & LG_VI(16825966-16820213) & POPTR_0006s18410 \\
\hline PoSetd7 & 468 & SETD & 2 & LG_IV(11613451-11616746) & gw1.IV.4590.1 \\
\hline PoSetd10 & 444 & SETD & 12 & LG_IV(7403229-7405799) & gw1.IV.3768.1 \\
\hline PoSetd6 & 439 & SETD & 1 & LG_VIII(2548612-2554748) & gw1.VIII.1187.1 \\
\hline PoSetd9 & 502 & SETD & 9 & LG_VII(9438694-9433649) & eugene3.01730002 \\
\hline PoSetd5 & 503 & SETD & 12 & LG_XIV(9526856-9530848) & eugene3.00141165 \\
\hline PoSetd1 & 551 & SETD & 4 & LG_XIV(2038778-2044599) & gw1.XIV.1045.1 \\
\hline PoSetd2a & 490 & SETD & 7 & LG_VIII(5640809-5644314) & gw1.VIII.2559.1 \\
\hline PoSetd2b & 345 & SETD & - & LG_X(15047671-15051672) & gw1.X.1011.1 \\
\hline
\end{tabular}

* Represent the subfamilies shown in Figure 1

genes were not proportional to the sizes of the predicted gene sets.

\section{Phylogeny and gene structures of Populus SET genes}

To understand the evolution of Populus SET genes, we performed unrooted phylogenetic analyses on the 106 SET genes from Populus (59 genes) and Arabidopsis (47) using Maximum Likelihood (ML), Bayesian inference (BI) and Neighbour-Joining (NJ) methods (Figures 1A and Additional file 1). The tree topologies produced by the three methods are largely consistent, with only minor differences at interior nodes (Additional file 1). The NJ tree is shown in Figure 1A and discussed below. Most Populus SET genes clustered with their homologs in Arabidopsis. Sometimes, however, two Populus genes clustered together with either a single Arabidopsis gene, or without any corresponding gene in Arabidopsis (Figure 1A, pink single brackets followed by numbers). In total, there are 19 such pairs of Populus SET genes.

The phylogenetic tree topology and the predicted protein domain compositions also allow the grouping of the 106 SET genes in Arabidopsis and Populus into eight subfamilies (named Suv, Atxr5, Ash, Atxr3, E(z), Trx, SMYD and SETD; Figure 1A), generally in accordance with those in other plants $[14,16-18]$. Nevertheless, there were some differences from prior classifications [16-18]. One notable difference is that recent classifications placed $M E A, C L F$ and $S W N$ in class I and ATX1, ATX2, ATX3, ATX4, $A T X 5, A T X R 3$ and ATXR7 in Class III [14-16], but our results had all of these genes clustering together, forming a monophyletic clade with bootstrap support of $77 \%$ (Figure 1A). In addition, each subfamily formed a group with high bootstrap support in the unrooted ML/BI/NJ analysis and may have the same ancestral origin.

In general, members from the same subfamily shared similar exon/intron structures, e.g. intron number and exon length; however, some members of the Suv, SMYD and SETD subfamilies (Figure 1B) had structural differences from other members. In the Suv subfamily, retrotransposition events have been reported $[17,18]$, which could have contributed to the diversity of the subfamily members. For subfamilies SMYD and SETD, we observed considerable diversity in gene structure and highly divergent sequences among subfamily members, but this diversity and its possible functions have rarely been reported.

\section{Expansion and evolution of the SET gene family in Populus}

The phylogenetic analysis of Populus SET genes indicates that they have experienced multiple gene duplication events. Gene duplication mechanisms include tandem duplication and large segmental/whole-genome duplication (WGD). To examine the relative contribution of each of these mechanisms in the expansion of the SET gene family, each member was electronically mapped to loci across all 16 Populus chromosomes and 4 additional scaffolds according to the location information provided by the JGI database [22] (Figure 2A). Chromosomes LG XI and LG XIX did not contain any SET genes (Figure 2A). The highest number of SET genes (7, or $11.9 \%$ of the total) was found on chromosome II, V and XIV (Figure 2A). Intriguingly, we did not identify gene clusters on any Populus chromosomes (Figure 2A), indicating that tandem duplication was not the cause of the detected duplicates in the Populus SET gene family. This is similar to the lack of tandem duplication events in the Arabidopsis and rice SET gene families [15].

Previous studies showed that the Populus genome contains evidence for three or more distinct WGD events $[22,25]$. Actually, strong support has been reported for two ancient WGD events in ancestral lineages shortly before the diversification of extant seed plants (255-400 Mya, million years ago) and extant angiosperms (150-275 Mya), respectively [25]. We analysed all of the duplicated 


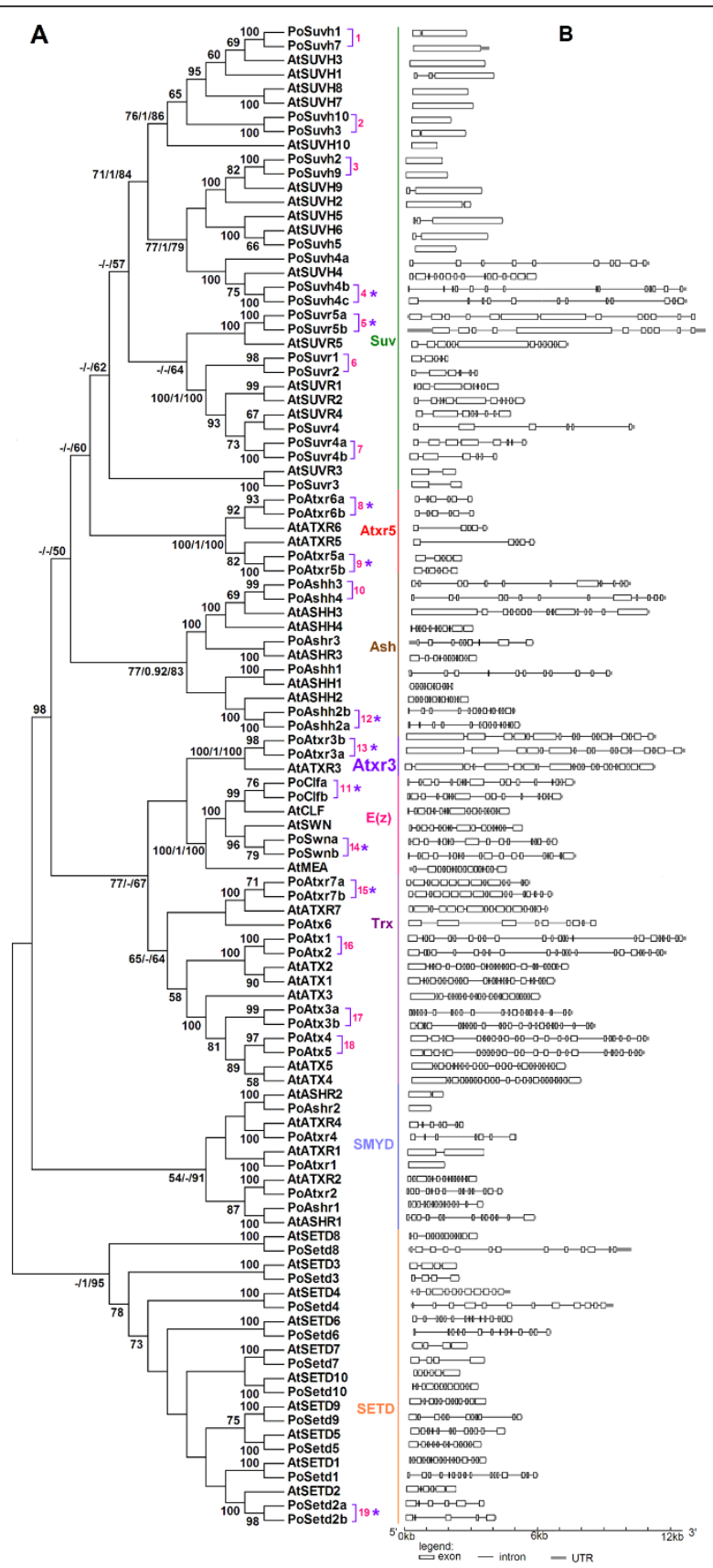

Figure $1 \mathrm{~A}$ phylogenetic tree and a schematic diagram for intron/exon structures of SET genes in Populus and Arabidopsis. (A) Unrooted tree constructed with NJ methods using SET domain amino acid sequences from Populus and Arabidopsis SET proteins. ML bootstrap values, Posterior probabilities from Bl and NJ bootstrap values analyses are presented for each main clade. ML and NJ bootstrap values above $50 \%$, and BI posterior probabilities greater than 0.90 analyses are shown. "-" represents the NJ or ML bootstrap values below 50 or the posterior probabilities low 0.90. There are eight subfamilies. The pink brackets followed by numbers denote duplicated gene pairs in Populus. (B) The intron-exon structure of the SET gene family in Populus and Arabidopsis.

gene pairs with intragenome syntenic relationships in the Populus genome from Plant Genome Duplication Database (PGDD) [23,26]. All 19 of the duplicated pairs of Populus SET genes resided in Populus segmental duplicated blocks (Figure 2A, gene pairs connected with lines; Table 2 and Additional file 2). Particularly, the Atx $4 / A t x 5$ and $A t x 3 a / A t x 3 b$ pairs were also located on two pairs of duplicated segmental blocks (Figure 2D and Additional file 2).

In addition, we calculated the values of synonymous substitutions per synonymous site $\left(K_{s}\right)$ of duplicated gene pairs from PGDD, which we assumed to be 

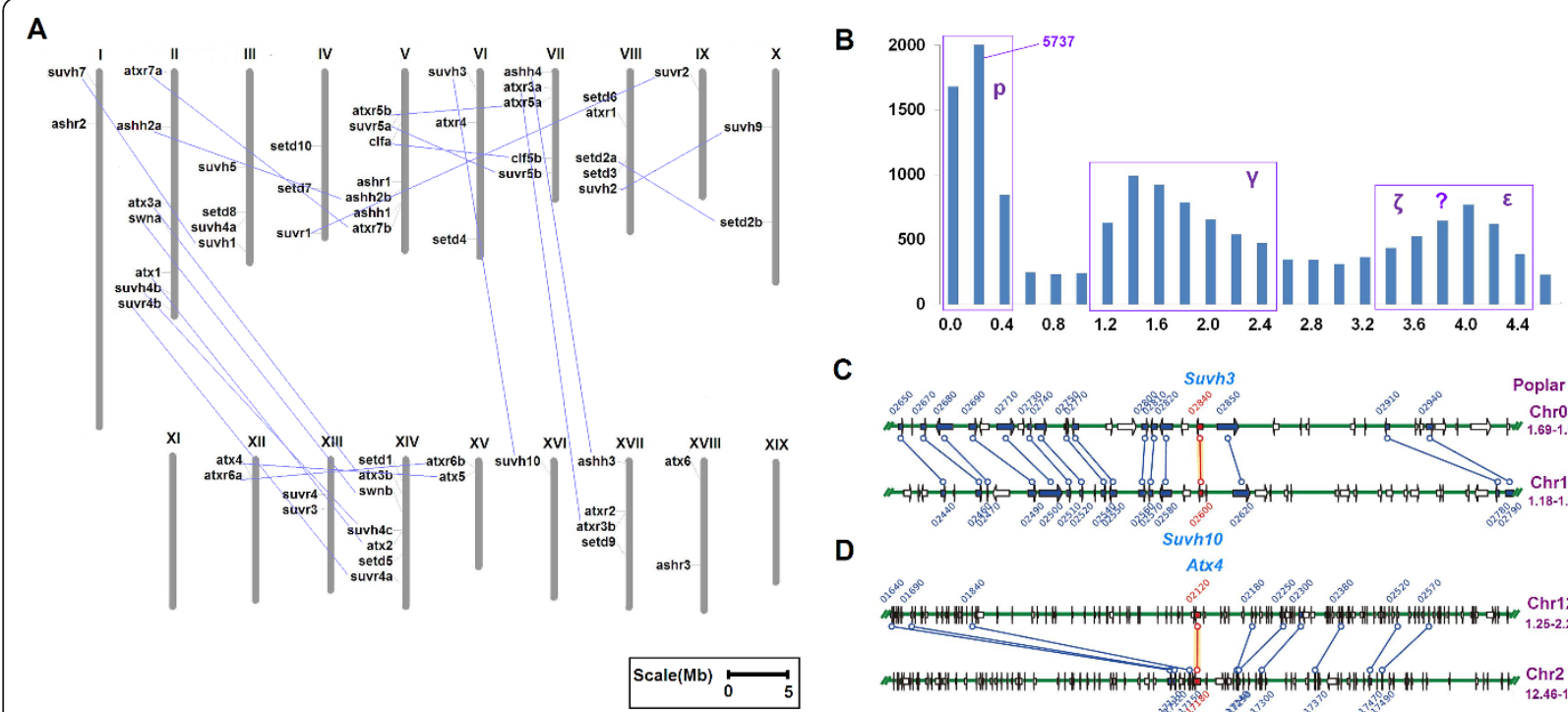

C

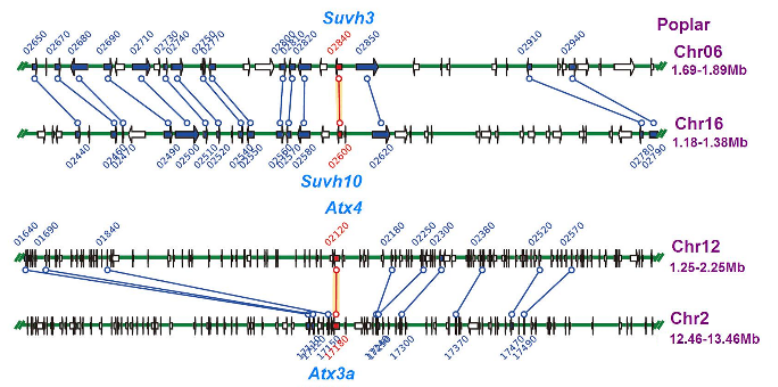

Figure 2 Positions of SET genes on Populus chromosomes. (A) Map of the SET gene positions on Populus chromosomes. Numbers refer to locus ID as listed in Table 1. The SET genes found on duplicated chromosomal segments are connected by lines. (B) Frequency distribution of $K_{s}$ between all syntenically duplicated gene pairs in Populus. The light blue box indicates a recent polyploidy event (since the split from the Arabidopsis lineage), denoted $p$; the pink box marks a duplication event apparently shared among the eurosids $(\gamma)$; the dark blue box denotes a

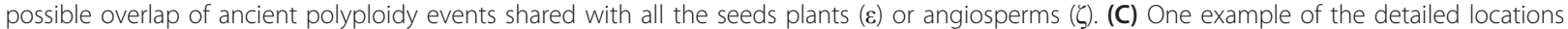
of representative pairs of genes duplicated in recent polyploidy events in the syntenic region. (D) Detailed location of representative duplicated pairs from ancient polyploidy events in the syntenic region.

correlated with the time of divergence since the genome duplication (Figure 2B). Apparently, the Populus duplicates found in syntenic blocks matched with two WGDs (the first and second peaks, denoted $p$ and $\gamma$ previously [23]). There was also a third peak $\left(K_{s}, 3.6-4.2\right.$, denoted $\varepsilon$ or $\zeta)$, which might be due to the ancient angiospermwide and/or seed plant-wide WGDs [25], with possible blurring of the distinction between the two expected peaks due to subsequent $K_{s}$ rate variation. To estimate the divergence time of the 19 duplicated gene pairs, we calculated their syntenic $K_{s}$ values. They could be classified at least into two categories (Table 2). The first category included the 19 gene pairs, whose locations in the syntenic region are shown in Figure $2 \mathrm{C}$ and Additional file 2 . They had an average $K_{s}$ value between 0.24830.2898 , corresponding to the first peak $(p)$ in Figure $2 \mathrm{~B}$. This duplication event was dated to 13.64-15.93 Mya, corresponding to a recent segmental duplication/WGD event in Populus, approximately 10-20 Mya after the split from the lineage leading to Arabidopsis [22]. The other category included one pair of duplicated blocks (between Atx 4/Atx 5 and Atx3a/Atx3b); their detailed locations in the syntenic region are shown in Figure 2D and Additional file 2. Interestingly, they had much higher average $K_{s}$ value, 1.5129 (Table 2), corresponding to the second peak. These duplicated blocks could be due to the retention of genes from an ancient WGD event(s) shared by core eudicots [23]. In addition the $K_{s}$ values of between Suvh4a and Suvh4b or Suvh4c were 1.6609 or 1.5428 , respectively (Additional file 3 : Table S1), suggesting that the duplication of Suvh4a and Suvh4b/c could be due to the WGD shared by the core eudicots. Other pairs of poplar $S E T$ genes have $K_{s}$ values ranging from 3.6 and 4.6, consistent with them being from even older WGD duplications, but the $K_{s}$ values are not reliable to assign the specific WGD. Our analysis strong suggests that segmental duplication events, especially those resulting from recent polyploidy events, have contributed to the expansion of the SET domain gene family in Populus.

\section{Domain diversity in Populus SET gene family}

Domains are basic functional and structural modules in proteins, and new combination of domains is associated with specific changes in protein functions $[27,28]$. We analysed the domain architecture of the $S E T$ gene family in Populus. In addition to the SET domain, most of these SET proteins included other domains with known or predicted functions. In particular, each of the six subfamilies found in our analysis had its own specific functional domains (Figure 3), similar to those in Arabidopsis, maize and rice $[15,16]$. In some subfamilies, there were gains and/or losses of domains. PreSET, SET, and PostSET are considered to be primordial domains [29], and 
Table 2 Estimated dates of the duplication events leading to pairs of SET genes in Populus and Arabidopsis

\begin{tabular}{|c|c|c|c|c|c|c|}
\hline \multirow{2}{*}{$\begin{array}{l}\text { Species } \\
\text { Populus }\end{array}$} & \multicolumn{2}{|c|}{ Duplicated pair } & \multirow{2}{*}{$\begin{array}{l}\text { Number of anchors } \\
777\end{array}$} & \multirow{2}{*}{$\frac{\text { Mean } K_{s}}{0.2674}$} & \multirow{2}{*}{$\begin{array}{l}\mathrm{SD} \boldsymbol{K}_{\boldsymbol{s}} \\
0.0922\end{array}$} & \multirow{2}{*}{$\begin{array}{l}\text { Estimated time (Mya) } \\
14.69\end{array}$} \\
\hline & Suvh2 & Suvh9 & & & & \\
\hline & Suvh3 & Suvh10 & 84 & 0.2841 & 0.1030 & 15.61 \\
\hline & Suvh4b & Suvh4c & 14 & 0.2566 & 0.0642 & 14.10 \\
\hline & Suvh7 & Suvh1 & 105 & 0.2597 & 0.0715 & 14.27 \\
\hline & Suvr1 & Suvr2 & 176 & 0.283 & 0.0956 & 15.55 \\
\hline & Suvr4a & Suvr4b & 15 & 0.2898 & 0.0978 & 15.93 \\
\hline & Suvr5a & Suvr5b & 19 & 0.2686 & 0.0968 & 14.76 \\
\hline & Atxr5a & Atxr5b & 118 & 0.2737 & 0.0927 & 15.04 \\
\hline & Atxr6a & Atxr6b & 46 & 0.2657 & 0.0875 & 14.60 \\
\hline & Ashh2a & Ashh2b & 381 & 0.2683 & 0.0894 & 14.74 \\
\hline & Ashh4 & Ashh3 & 16 & 0.2568 & 0.0723 & 14.11 \\
\hline & Atxr3a & Atxr3b & 109 & 0.2758 & 0.0877 & 15.15 \\
\hline & Clfa & Clfb & 29 & 0.2844 & 0.0966 & 15.62 \\
\hline & Swna & Swnb & 394 & 0.2697 & 0.0842 & 14.82 \\
\hline & Atx1 & Atx2 & 11 & 0.2483 & 0.0665 & 13.64 \\
\hline & Atx3a & Atx3b & 394 & 0.2697 & 0.0842 & 14.82 \\
\hline & Atx4 & Atx5 & 46 & 0.2657 & 0.0875 & 14.60 \\
\hline & Atxr7a & Atxr7b & 566 & 0.2517 & 0.0548 & 13.83 \\
\hline & Setd2a & Setd2b & 1034 & 0.2594 & 0.0583 & 14.09 \\
\hline & Atx4/5 & Atx3a/b & 46 & 1.5129 & 0.1836 & 83.13 \\
\hline \multirow[t]{5}{*}{ Arabidopsis } & SUVH3 & SUVH7 & 177 & 0.8413 & 0.2201 & 28.04 \\
\hline & SUVR2 & SUVR1 & 33 & 0.8419 & 0.2180 & 28.06 \\
\hline & SWN & MEA & 41 & 0.8182 & 0.1871 & 27.27 \\
\hline & ATX1 & ATX2 & 113 & 0.8635 & 0.2162 & 28.78 \\
\hline & $\mathrm{ASHH} 4$ & ASHH3 & 138 & 0.8422 & 0.2319 & 28.07 \\
\hline
\end{tabular}

The mean $K_{s}$ value was calculated for each pair of protein-coding genes within a duplicated block and used to date the duplication events. $K_{s}$ values of all duplicated gene pairs greater than 1.5 and less than 0.5 were discarded for Arabidopsis; $K_{s}$ greater than 0.4 for Populus was also discarded, except Atx $4 / 5$ and Atx3a/b

they are usually organized in proteins in the order PreSET/SET/PostSET, as seen in the Suv subfamily. Other domains (such as SAR, ZnF_C2H2 and WIYLD) were integrated into this primordial structure to form new gene family members; other members lost one or more of the primordial domains during their evolution (Figure 3 ). The general patterns of domain gains and losses were similar in the Ash, Trx and E(z) subfamilies.

Some duplicated gene pairs (8/19) also experienced some domain gains and losses (Figure 3, in the black box, and Table 3). For instance, Suvr5b gained a ZnF_C2H2 motif near the N-terminus of the encoded protein compared with its counterpart Suvr5a (Table 3). Compared with its counterpart Atxr $7 a, A t x r 7 b$ lost the Pfam: Luteo_coat domain near the $\mathrm{N}$ terminus (Table 3). We have checked all 5181 pairs of genes produced by the most recent rounds of WGD in Populus, and found that 783 pairs of them (only 15\%) have experienced domain gain and losses. In contrast, among the 18 pairs of WGD duplicates in the SET domain gene family, $\sim 45 \%$ of them (8 pairs) have experienced domain gain and losses (Fisher's Test, p-value $=0.016$ ). These gains and losses of domains tended to occur near the $\mathrm{N}$ terminus $(6 / 8)$, although they were occasionally found at the $C$ terminus (two pairs: Suvh4b and Suvh4c, Suvr4a and Suvr4b). Most SET domains are located near the $C$ terminus and there might be specific functional constraints that protect the stability of the domain architecture at the $\mathrm{C}$ terminus.

The analysis of the Populus SET genes indicated that one of two recent SET duplicate undergoes domain gain or loss, during a relatively short period of evolutionary time following a recently WGD event. New domain architectures can drive the evolution of organismal complexity [30]; for example, recombination of domains encoded by genes belonging to the yeast mating pathway had a major influence on phenotype [31]. Therefore, the domain gains and losses in SET genes that occurred 13.64-15.93 Mya might have been a strong force of evolution of Populus complexity. Because SET 


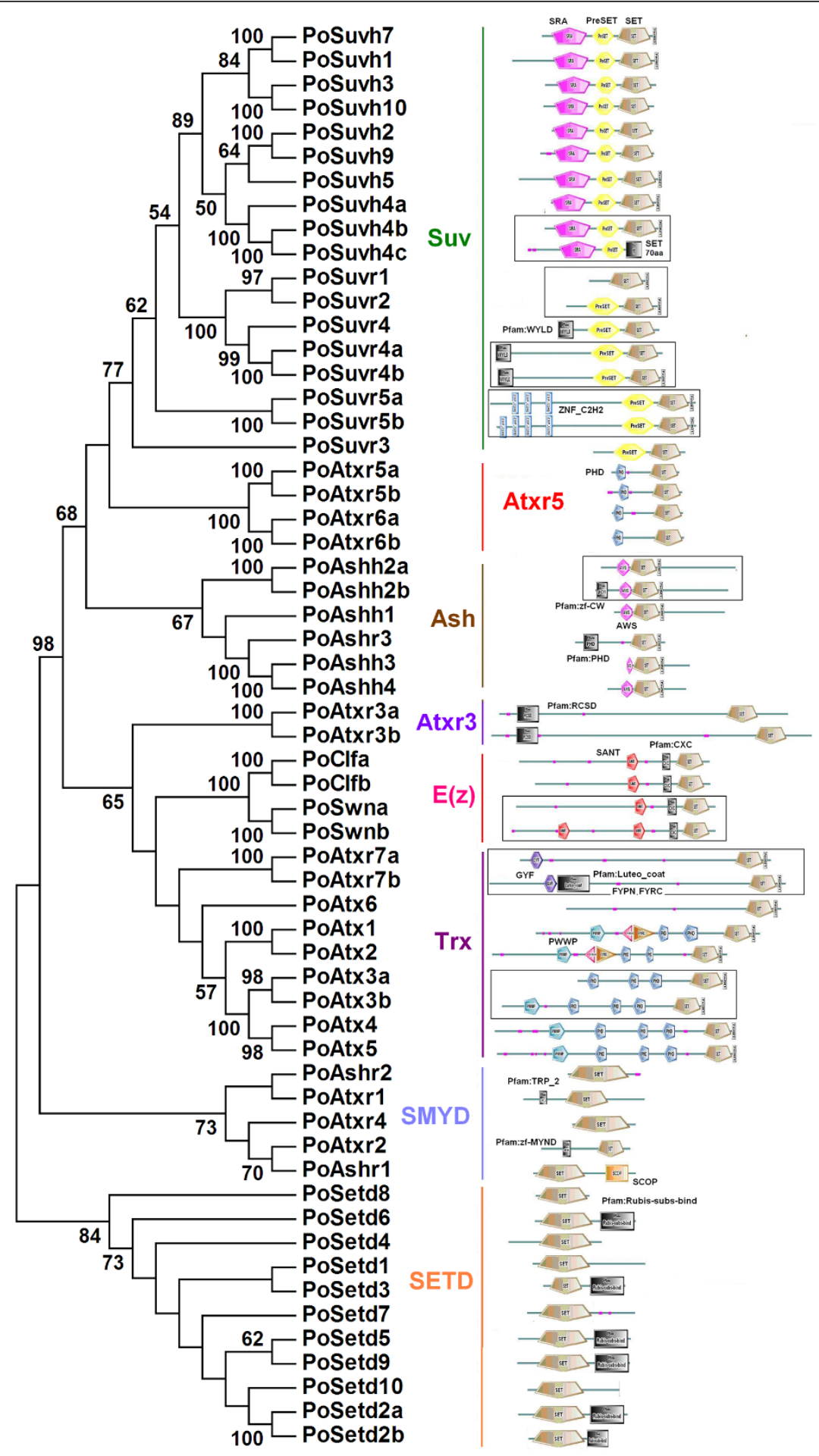

Figure 3 An unrooted NJ tree using SET domain amino acid sequences from Populus SET genes (A) and the domain architecture of SET proteins (B). The tree depicts eight subfamilies based on phylogenetic relationship are given as in Figure 1. The black box indicates duplicated gene pairs with domain gains and losses.

proteins are important for histone modification and chromatin structure, they can play crucial roles in regulating gene expression during plant development [6,32].
That their domain architecture has incurred major changes in a short time indicates that epigenetic regulation could be somewhat plastic. 
Table $3 K_{a} / K_{s}$ and divergence analysis of domain architecture and gene structure of duplicate d SET gene pairs in Populus

\begin{tabular}{|c|c|c|c|c|c|c|c|}
\hline \multicolumn{2}{|c|}{ Duplicated pairs } & \multirow{3}{*}{$\begin{array}{l}K_{a} / K_{s} \\
0.09\end{array}$} & \multicolumn{3}{|c|}{ Domain gain/lost } & \multicolumn{2}{|c|}{ The terminus diversity } \\
\hline & & & \multirow{2}{*}{ Numbers } & \multirow{2}{*}{$\begin{array}{l}\text { Name } \\
/\end{array}$} & \multirow{2}{*}{$\frac{\text { Location }}{/}$} & \multirow{2}{*}{$\begin{array}{l}\mathbf{5}^{\prime} \text {-terminal } \\
1 \mathrm{~A}^{* *}, 2 \mathrm{~A}, 4\end{array}$} & \multirow{2}{*}{$\begin{array}{l}3 \text { '-terminal } \\
4\end{array}$} \\
\hline Suvh2 & Suvh9 & & & & & & \\
\hline Suvh3 & Suvh10 & 0.17 & / & / & / & 3,4 & 4 \\
\hline Suvh4b & Suvh4c & 0.14 & 1 & PreSET & $C^{*}$ & $1 \mathrm{~A}, 4$ & 1B \\
\hline$\overline{S u v h 7}$ & Suvh1 & 0.24 & 1 & / & / & 3,4 & 4 \\
\hline Suvr1 & Suvr2 & 0.35 & 1 & PreSET & $\mathrm{N}$ & $1 \mathrm{~A}, 2 \mathrm{~A}$ & $1 \mathrm{~A}, 2 \mathrm{~A}$ \\
\hline Suvr4a & Suvr4b & 0.23 & 1 & PreSET & C & $1 \mathrm{~A}, 4$ & 4 \\
\hline Suvr5a & Suvr5b & 0.20 & 1 & $\mathrm{ZnF}-\mathrm{C} 2 \mathrm{H} 2$ & $\mathrm{~N}$ & $1 \mathrm{~A}, 2 \mathrm{~A}, 3,4$ & 4 \\
\hline Atxr5a & Atxr5b & 0.11 & / & / & / & $1 \mathrm{~A}, 4$ & 4 \\
\hline Atxr6a & Atxr6b & 0.19 & / & I & I & $1 \mathrm{~A}, 2 \mathrm{~A}$ & I \\
\hline Ashh2a & Ashh2b & 0.22 & 1 & Pfam:zf-CW & $\mathrm{N}$ & $1 \mathrm{~A}, 1 \mathrm{~B}, 2 \mathrm{~A}, 2 \mathrm{~B}, 4$ & $1 \mathrm{~A}, 1 \mathrm{~B}, 4$ \\
\hline Ashh4 & Ashh3 & 0.18 & / & / & / & 4 & 4 \\
\hline Atxr3a & Atxr3b & 0.28 & / & I & I & $1 \mathrm{~A}, 2 \mathrm{~A}, 4$ & 4 \\
\hline Swna & Swnb & 0.26 & / & 1 & / & $1 \mathrm{~A}, 2 \mathrm{~A}, 3,4$ & 4 \\
\hline $\mathrm{Clfa}$ & $\mathrm{Clfb}$ & 0.18 & 1 & SANT & $\mathrm{N}$ & $1 \mathrm{~A}, 1 \mathrm{~B}, 2 \mathrm{~A}$ & 4 \\
\hline Atx1 & Atx2 & 0.19 & I & I & / & $1 \mathrm{~A}, 2 \mathrm{~A}, 2 \mathrm{~B}, 4$ & 4 \\
\hline Atx3a & Atx3b & 0.37 & 1 & PWWP & $\mathrm{N}$ & $1 \mathrm{~A}, 2 \mathrm{~A}, 4$ & 4 \\
\hline Atx4 & Atx5 & 0.30 & I & I & 1 & $1 \mathrm{~A}, 2 \mathrm{~A}, 4$ & $1 \mathrm{~B}, 4$ \\
\hline Atxr7a & Atxr7b & 0.52 & 1 & Pfam:Luteo_coat & $\mathrm{N}$ & $1 \mathrm{~A}, 2 \mathrm{~A}, 4$ & $1 \mathrm{~B}$ \\
\hline$\overline{S e t d 2 a}$ & Setd $2 b$ & 0.39 & / & / & / & $1 \mathrm{~B}, 3,4$ & $1 \mathrm{~A}$ \\
\hline
\end{tabular}

*: N: N-terminus of protein, C: C-terminus of protein. ${ }^{* *}$ : Shown in Figure 4

\section{Expression analysis of SET genes in Populus}

To learn about the expression patterns of SET genes, we reanalysed the Populus microarray data generated by Wilkins and co-workers [33]. Only four SET genes (Suvh1, Atx6, Suvr5a and Clfa) did not have corresponding probes in that dataset, and the expression profiles of the other 55 SET genes were analysed as shown in Figure 5 and Additional file 4. We also investigated the frequency of ESTs from EST databases at National Center for Biotechnology Information (NCBI) (March, 2011) and obtained digital expression profiles of 47 Populus SET genes; the other 14 SET genes did not have EST data (Table 1). The SET genes were expressed widely in a number of tissues; intriguingly, expression level of the $S E T$ genes in specific tissues was higher in young leaves (YL) than in other tissues ( $\mathrm{t}$-test, $p$-values $<0.05$; Additional file 3: Table S2), except the mature leaves (ML) and roots $(\mathrm{R})$. In contrast, the expression level in differentiating xylem (DX) was lower than in other tissues (t-test, $p$-values < 0.05; Additional file 3: Table S2), except light-grown seedling $(C L)$ and dark-grown seedlings (DS).

Based on hierarchical clustering, the expression patterns of SET genes could be divided into four groups: G1-G4 (Figure 5 and Table 4). G1 can be divided into two subgroups: G1-Sub1 and G1-Sub2 (Figure 5). Their expression patterns are listed in Table 4. The ortholog of $C l f b$ in Arabidopsis was CLF, which is required to repress FLC [10]. Based on the expression of Clfb in Populus, we inferred that $C l f b$ could have a similar effect on flowering and the shape of mature leaves in Populus.

In addition, we determined the expression profiles of the duplicated SET gene pairs. In 12 of the 19 duplicated gene pairs, both copies were co-expressed (values above 100 are considered to be expressed). As shown in Figure 6, the expression profiles of these 12 duplicated SET genes formed two patterns: i) one paralog was expressed higher than the other in at least one tissue, whereas the other was expressed at a higher level in some other tissues (Figure 6, purple box); or ii) one paralog had lower expression levels than the other in all tissues (Figure 6, black box).

\section{Structural divergence of duplicated Populus SET gene pairs via four major scenarios}

Previous studies showed that structural divergences have played important role during the evolution of duplicates in plants $[34,35]$. To understand the structural divergence of duplicated Populus SET gene pairs, we compared gene structures between the two recent paralogs in each of the 19 duplicated gene pairs. We found that the 3'-and 5'- 
terminal regions (often containing a portion of the coding region) of most duplicated Populus SET pairs were highly divergent (Table 3 and Additional file 5). The 5'-terminal end is more polymorphic than the 3'-terminal end (MannWhitney U-test, p-value $=0.00012$, Table 3). Further investigation of their sequences revealed that these gene structure changes could have originated from one (or more) of four major scenarios (Figure 4).

We have found that the 3'-terminal and 5'-terminal regions (often with part of the coding region) of most duplicated Populus SET gene pairs were highly divergent, particularly the 5 ' end (Figure 4 and Additional file 5). Further investigation of their sequences revealed that these gene structure differences could have originated from one (or more) of four major scenarios (Figure 4). The first scenario is insertion or deletion (indel) of partial or entire exons (Figure 4-1A and 6-1B). For example, the first exons at the 5' ends of Atxr7a and Atxr $7 b$ matched very well with one other except that the exon in $A t x r 7 b$ lacked three nucleotides at its beginning, indicating an indel event of one codon (Additional file 6 -e.g. 1A). A notable indel case was the loss of an entire exon at the 5' end of the Clfb gene in comparison with Clfa (Additional file 6 -e.g. 1B). Changes in exon length between most duplicated pairs appeared to have resulted from this scenario (Figure 4-1A \&4-1B and Table 3).

The second scenario was the intronization/exonization of a partial or entire exon/intron (Figure 4-2A \&4-1B). The second exons at the 5 ' ends of Clfa and Clfb were extremely similar in sequence, but Atxr $7 a$ was about 300 bp longer at its 3' end; we found that part of a Clfb intron had been exonized in $\mathrm{Clfa}$ (Additional file 6 -e.g. 2A). In another interesting case, the second exon in Ashh2a matches an intronic region in $A \operatorname{shh} 2 b$, demonstrating intronization of the whole exon (Additional file 6 -e.g. 2B).

The third scenario was the gain of introns, which divide one exon into two or more smaller ones, or the loss of introns, which unite two or more exons into one longer one (Figure 4-3). The exons near the 5' ends of Suvh7 and Suvh1 make a persuasive example (Additional file 6 -e.g. 3). There were two exons, 305 and 1657, at the 5' end of Suvh1, which flanked a 59 bp intron; whereas there was a single exon, 2010, at the 5' end of Suvh7, without an intron.

The last scenario involved an increase or decrease in the UTR at the 5' ends of duplicated pairs genes (Figure 4). Generally, the UTR of one duplicate was longer than the other. For example, Suvh3 had a longer UTR at its 5' end than its corresponding region in Suvh10 (Additional file 6 -e.g. 4). Taken together, Scenarios $1 \mathrm{~A}$ and $2 \mathrm{~A}$ alters exon length, whereas Scenarios $1 \mathrm{~B}$ and $2 \mathrm{~B}$ and 3 change exon number, or scenario 3 change intron number. In contrast, the 3' ends of the duplicates were not as different as the 5' ends. The most common case was a change in the length of the UTR at the 3' end.

\section{Discussion}

We have performed systematic phylogenetic analysis of Populus SET genes, and determine $K_{s}$ values of duplicates and examined their expression patterns. Our analyses indicate that $S E T$ genes have experienced many duplication events during the Populus evolutionary history. The relatively recent duplicates are likely the results of whole-genome duplication, and many show structural divergence, including gain/loss of functional domains. Furthermore, at least some recent paralogs exhibit divergent expression patterns. Therefore, SET genes in Populus provide a case study for evolution of genes following duplication, showing both gene loss and retention with functional divergence at both structural and expression levels.

\section{Genome duplication and evolution of SET genes}

There are 59, 47 and 43 known SET genes in Populus, Arabidopsis and rice, respectively. The Populus genome has experienced at least four rounds of genome duplication: two ancient duplication events prior to the gymnosperm-angiosperm divergence and before the diversification of all extent angiosperms, an intermediate event shared with all core eudicots plants, and a recent event occurring after the divergence of the lineages leading to Populus and Arabidopsis [22,23,25]. Our analysis suggested that, of the 19 duplicated pairs of adjacent Populus SET genes on the phylogenetic tree, all resulted from the recent WGD event. Among older duplicates prior to the split of Populus and Arabidopsis, one or two Suv pairs and one Atx pair likely resulted from the core eudicot WGD, and possibly others from older WGDs. Given that at least four rounds of WGD are known in the Populus lineage, many genes loss within the SET gene family appears to have occurred in Populus. The Arabidopsis lineage has experienced two WGD events since its split from the Populus lineage and has lost many of the duplicates [36]. Our investigation of Arabidopsis SET genes revealed five duplicated gene pairs since its divergence from Populus. These five pairs had average $K_{s}$ values between $0.1817-0.2319$, with estimated dates between 27.27-28.78 Mya for the duplication, corresponding to a recent WGD in Arabidopsis [22,23]. Furthermore, soybean has experienced one more WGD event than Populus since its split with the Populus lineage, and it has 25 more SET genes than Populus. Most of the increased soybean $S E T$ gene number resulted from the recent WGD (data not shown). Therefore, the expansions in the SET gene family in Arabidopsis, Populus, and soybean could be explained by gene loss and gain after WGD events.

An increase in the number of regulatory genes (i.e. transcriptional and developmental regulators) is one of important factors that facilitated the evolution of more 


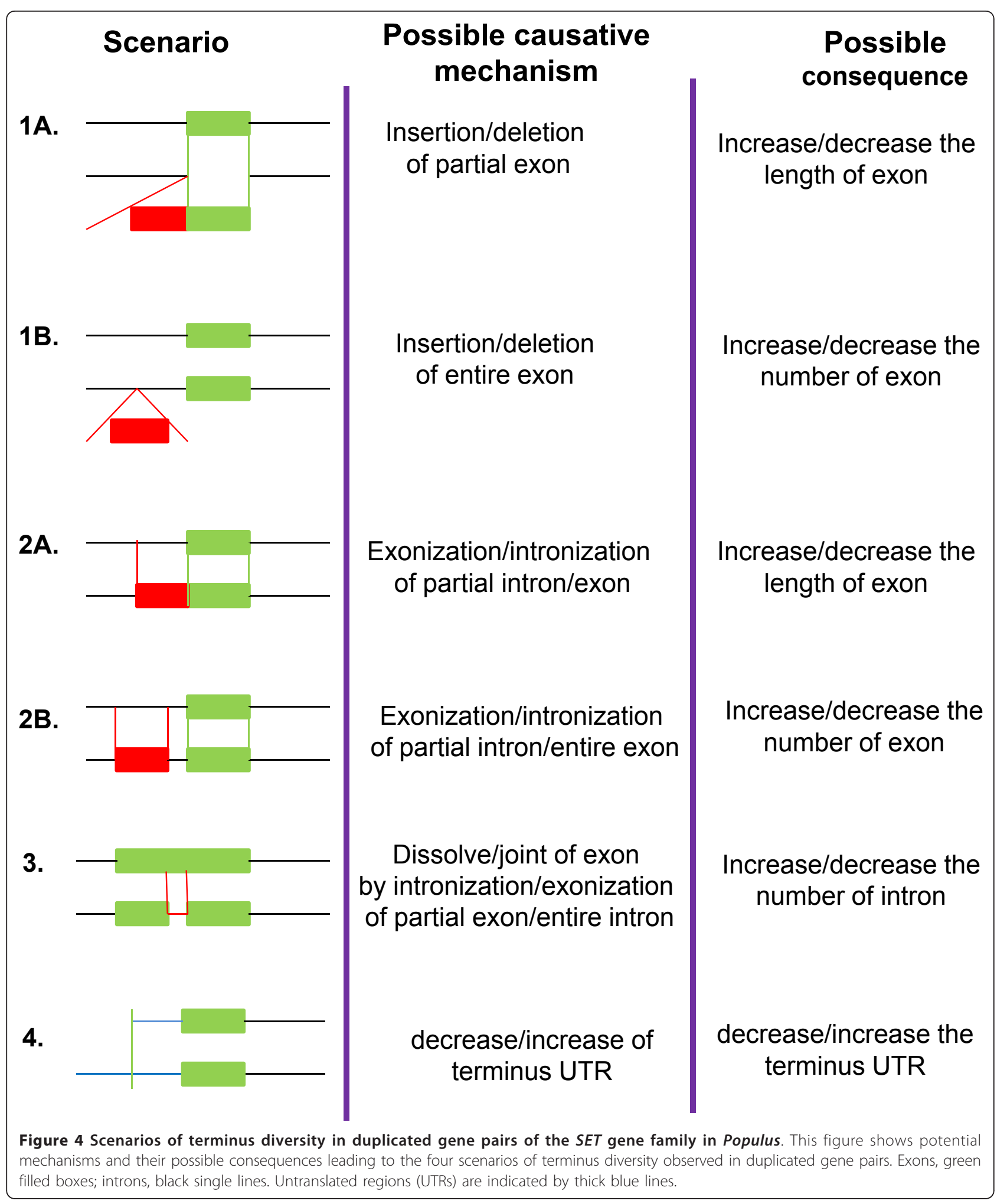

complex developmental systems [36]. Maere et al. estimated that more than $90 \%$ of the increases in Arabidopsis regulatory genes were likely caused by genome duplications during the last 150 million years [37]. Our results suggest that WGD could be the main mechanism for the expansion of the SET gene family in Populus, consistent with this idea. To date, many studies have shown that many members of transcriptional factor (TF) gene 


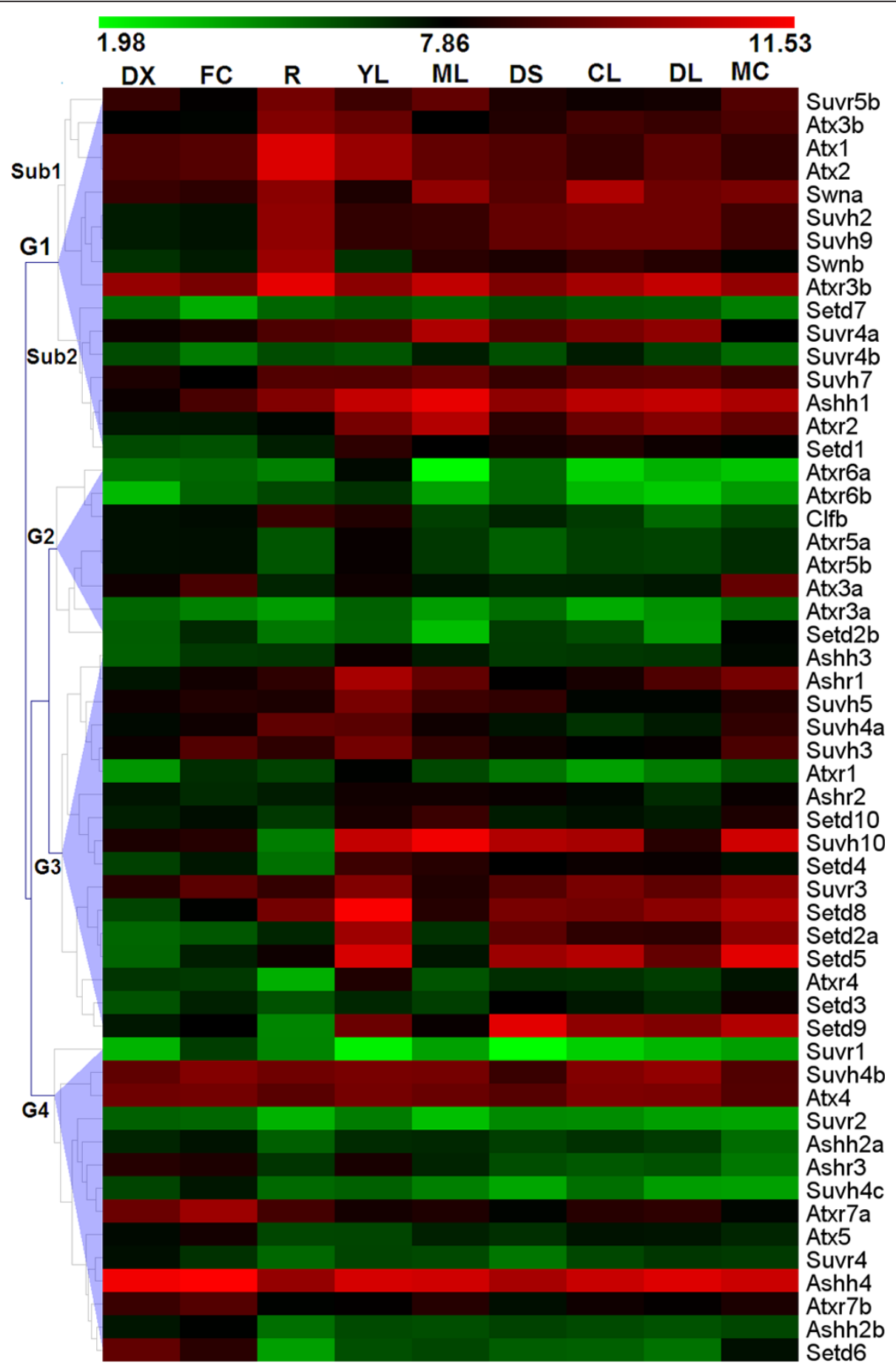

Figure 5 Hierarchical clustering of expression profiles of Populus SET genes across different tissues, organs and treatments. The expression data were gene-wise normalized and hierarchically clustered based on the Pearson correlation. The relative expression levels of genes in each row are normalized against the maximum value. Colour scale at the top of each dendrogram represents log2 expression values, green represents low levels and red indicates high levels of transcript abundances. $\mathrm{CL}$, continuous light-grown seedling; $\mathrm{DL}$, etiolated dark-grown seedling transferred to light for 3 h; DS, dark-grown seedlings; YL, young leaf; ML, mature leaf; R, root; DX, differentiating xylem; FC, female catkins; MC, male catkins. G1-Sub1, G1-Sub2, G2, G3, G4 represent different subgroup. 
Table 4 The expression patterns of SET genes

\begin{tabular}{|c|c|c|}
\hline Groups & Members & Expression patterns \\
\hline \multirow[t]{2}{*}{$\begin{array}{l}\text { G1- } \\
\text { Sub1 } ※\end{array}$} & $\begin{array}{l}\text { Suvr5b/2/9, Swna/ } \\
\text { b, }\end{array}$ & All showed high expression level in all tissues except Swnb showed relatively low expression in FC and $\mathrm{YL}$ \\
\hline & $\begin{array}{l}\text { Atx } 1 / 2, \text { Atx3b, } \\
\text { Atxr3b }\end{array}$ & \\
\hline \multirow[t]{2}{*}{ G1-Sub2 } & $\begin{array}{l}\text { Suvr4a/b, Suvh7, } \\
\text { Ashh1, }\end{array}$ & $\begin{array}{l}\text { Most are expressed at lower levels and with different patterns than the first subgroup. Setd7 and Suvr4a have low } \\
\text { expression levels in all the tissues. }\end{array}$ \\
\hline & Atxr2, Setd7/1 & \\
\hline \multirow[t]{2}{*}{ G2 } & $\begin{array}{l}\text { Atxr5a/5b/6a/6b, } \\
\text { Atx3a, }\end{array}$ & $\begin{array}{l}\text { Most had very low expression levels in almost all tissues, but the Clfb and Atx3a expressed at higher levels in certain } \\
\text { tissues. }\end{array}$ \\
\hline & $\begin{array}{l}\text { Atxr3a, Clfb, } \\
\text { Setd2b }\end{array}$ & \\
\hline \multirow[t]{3}{*}{ G3 } & $\begin{array}{l}\text { Suvh3/4a/5/10, } \\
\text { Suvr3, }\end{array}$ & $\begin{array}{l}\text { Most had high expression levels in } \mathrm{YL}, \mathrm{ML} \text { and others, but low expression in FC. Suvr1, Atxr1 and Ashh3 have low } \\
\text { expression levels in all the tissues. }\end{array}$ \\
\hline & $\begin{array}{l}\text { Ashh3, Ashr2/1, } \\
\text { Atxr1/4, }\end{array}$ & \\
\hline & $\begin{array}{l}\text { Setd2a/3/4/5/8/9/ } \\
10\end{array}$ & \\
\hline \multirow[t]{2}{*}{ G4 } & $\begin{array}{l}\text { Ashh2a/2b/4, } \\
\text { Ashr3 }\end{array}$ & $\begin{array}{l}\text { Most genes had low expression levels in all the tissues, but Ashr3, Atx5, Setd6 and Atxr7b showed high expression } \\
\text { levels only in FC and MC }\end{array}$ \\
\hline & $\begin{array}{l}\text { Atxr7a/b, Atx4/5, } \\
\text { Setd6 }\end{array}$ & \\
\hline
\end{tabular}

※ased on hierarchical clustering as shown in Figure 5

families survive after WGD events [33,36,38], but few papers report on epigenetic regulatory gene families. SET genes are important epigenetic regulatory gene and have been largely retained after WGDs. Epigenetic regulators modulate expression of a large number of functionally related genes. Therefore, our study suggests that more basic regulatory gene families could have evolutionary mechanisms similar to TF genes, which might contribute to the evolution of gene networks and provide insight into chromatin regulatory evolution.

\section{Expression profiles of SET genes and functional diversity of duplicated pairs in Populus}

We investigated Populus microarray and EST data and found that most $S E T$ genes are expressed relatively widely, suggesting that the Populus SET genes that have survived after WGD events are likely functional. Similarly, almost all Arabidopsis and soybean SET genes were expressed (Additional file 3: Tables S3, S4), again suggesting that these $S E T$ genes have functions.

According to the expression patterns of Populus genes and the functions of their Arabidopsis orthologues, we could hypothesize possible functions of these genes in Populus. For example, the orthologue of Swnb in Arabidopsis is $S W N$, which is involved in H3K27 trimethylation at important floral and shoot developmental genes, including AGAMOUS and SHOOT MERISTEMLESS (STM) [39]. Therefore, Swnb might have a function similar to SWN in regulating Populus flower and shoot development. However, some SET genes were specific to Populus, for example, Suvr4a, Suvr4b, Atx3a, and Atx3b. All of these genes have abundant transcripts in Populus and are expressed at different levels in different tissues. These results suggest that their counterparts in Arabidopsis have been lost and the functions are either not needed in Arabidopsis or performed by other genes.

Interestingly, one of the greatest differences between the Populus SET genes and Arabidopsis SET genes is that there are 19 duplicated Populus SET gene pairs but only five Arabidopsis SET gene pairs. In 12 of the 19 duplicated gene pairs, both copies of were expressed, with two types of expression patterns.

The first type is that one copy was expressed at higher levels than the other in one or more tissues, but the other copy was higher in some other tissue(s) (Figure 6, purple boxes). For example, $A \operatorname{shh} 2 a$ and $A s h h 2 b$ corresponded to ASHH2 in Arabidopsis, which negatively regulates shoot branching [40]. The Ashh $2 a$ and Ashh $2 b$ duplicates in Populus are both expressed at the same stages and could repress shoot branching in Populus. Swna and Swnb corresponded to the Arabidopsis $S W N$, which exhibits partial functional redundancy with CLF and MEA [6]. Populus is a perennial woody plant with a juvenile-to-mature phase change, so its flowering processes is different from that of Arabidopsis and may require two duplicates with somewhat different functions to regulate these processes.

The second type is that one duplicate was expressed at higher levels than the other in all tissues (Figure 6, black boxes), suggesting that ther former has a stronger function than the latter. The Arabidopsis ATXR3 gene corresponds 


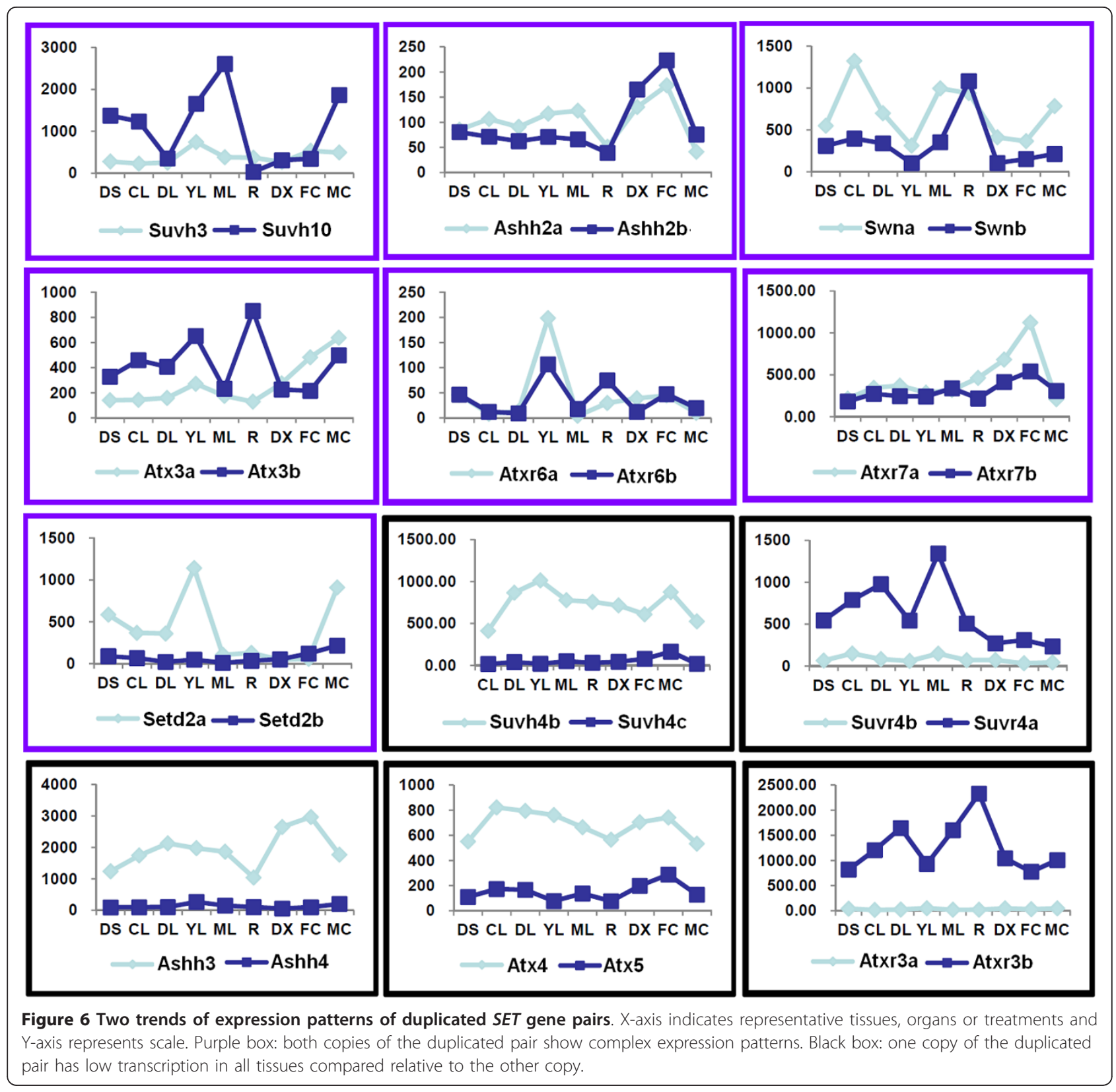

to the poplar Atxr3a and Atxr3b. ATXR3 mutants are smaller with curly leaves, short roots, early flowering, and female sterility $[12,13]$. The closest Populus homologues of ATXR3 may also play important roles in regulating broad developmental processes, consistent with one duplicate having wide-ranging high level expression. Similarly, the fact that Arabidopsis SUVH4 is responsible for the majority of H3K9 dimethylation in heterochromatin and affects the number of floral organs and the expression of $P H O S$ PHOANTHRINILATE ISOMERASE (PAI) [6] suggests that the Populus homologues Suvh4a and Suvh4b could also regulate multiple developmental processes.
The two different expression patterns in different tissues of the 12 duplicated gene pairs suggest functional diversification and possible function redundancy (or gene silencing), respectively. Many models for the evolution of gene duplications have been proposed in the past nearly four decades [41]. Recently, divergence in expression between duplicates has also been examined for Arabidopsis regulatory genes [42]. The first pattern may support the duplication-degeneration-complementation (DDC) model, in which divergent expression in different tissues allows functional differentiation of the duplicates and finally lead to subfunctionalization or neofunctionalization $[43,44]$. 
On the other hand, the latter pattern might suggest functional reduction for one copy (hypofunctionalization), before the weak copy is completely lost (nonefunctionalization) $[41,45,46]$.

\section{Conclusions}

We have shown that Populus has gained additional SET genes compared with its common ancestor with Arabidopsis, due to a WGD since the divergence from the lineage leading to Arabidopsis. Those duplicates that have been retained show divergence in both coding regions and expression levels, suggesting that the $S E T$ genes might have experienced functional diversification, including possible subfunctionalization. The increased number of $S E T$ genes with potentially distinct functions could have supported the evolution of epigenetic gene regulation of a woody perennial that is more complex than that of an herbaceous annual.

\section{Methods}

\section{Database search for SET genes}

The complete genome and predicted proteomes sequence of Populus was obtained from the JGI [http://genome.jgipsf.org/Poptr1_1/Poptr1_1.home.html] database [22]. To identify all genes for proteins containing SET domains in Populus, the SET domain PF00856 model from the Pfam database $[47,48]$ was used to perform a local search of the Populus predicted proteomes using the HMMER program (2.3.2) [49]. To find genes for similar proteins from unannotated genomic regions, the identified proteins sequences (domains) were used as queries for a gene search against the Populus genome sequence using a newly developed software, Phoenix (Protein Homologue Extraction, Sun et al., unpublished). An $e$-value cutoff value of 1e-5 was used for further analysis. SET genes from Arabidopsis were obtained as described by Zhang et al. [15].

\section{Multiple sequence alignment, gene structure and protein} architecture analyses

Multiple sequence alignment using MUSCLE with default parameters [50] was performed on SET proteins, and the alignment was subsequently adjusted manually. Gene structure information, including the intron/exon distribution pattern, was obtained by GSDS (Gene Structure Display Server), developed by CBI [51]. Structural motif annotation was performed using the SMART [http://smart.embl-heidelberg.de] and Pfam [http://pfam. sanger.ac.uk] databases.

\section{Genome localization and syntenic analysis of SET genes}

Information on chromosomal location was gathered from the Populus genome browser. MapInspect [http://www. plantbreeding.wur.nl/UK/software_mapinspect.html] was used to map the SET genes onto chromosomes. Syntenic information was collected from the Plant Genome Duplication Database [PGDD: http://chibba.agtec.uga.edu/ duplication] [23,26].

\section{Calculating $K_{s}$ and dating the duplication event}

Protein amino acid sequences of all the duplicated gene pairs from Populus were aligned using MUSCLE with default parameters [50], and the results were used to guide the alignments of DNA coding sequences (CDS) by Pal2Nal [52]. $K_{s}$ and $K_{a}$, the number of synonymous and nonsynonymous substitutions per site, were determined using the aligned CDS by yn00 in PAML 4.3 [53].

In dating segmental duplication events, the approximate date of the duplication event was calculated using the mean $K_{s}$ values from $\mathrm{T}=K_{s} / 2 \lambda$, where the clocklike rate $(\lambda)$ for Populus was $9.1 \times 10^{-9}$ [54]. Similarly, the average $K_{s}$ of all the duplicated SET gene pairs from Populus and Arabidopsis was calculated by the same method, where the clock-like rate $(\lambda)$ for Arabidopsis was $1.5 \times 10^{-8}$.

\section{Tree building}

Phylogenetic trees for the aligned SET protein sequences were constructed using NJ, ML, and BI methods. The NJ tree was constructed using MEGA [55] with the "pairwise deletion" option and "Poisson correction" model. Bootstrap support was estimated from 1000 replicates to evaluate the reliability of internal branches. ML trees were generated using PhyML version 3.0.1, with 100 nonparametric bootstrap replicates and WAG model [56]. MrBayes software [57] was used to construct BI trees, using the WAG model of evolution, after running for 1,000,000 generations, with four Markov chains sampled every 1000 generations.

\section{Gene expression analysis}

To search for SET genes from Populus ESTs, we used the Populus SET CDS as query sequences to search for highly similar ESTs sequences (at least $160 \mathrm{bp}$ and $95 \%$ identity) in Populus using the NCBI database. We also analyzed the public microarray dataset of Populus [33]; we normalized and hierarchically clustered them based on Pearson coefficients with average linkage in the $\mathrm{MeV}$ (version 4.8) program [58]. Furthermore, we also got the RNA-seq dataset of soybean [59].

\section{Additional material}

Additional file 1: Unrooted phylogenetic trees constructed using (A) ML and (B) BI methods based on SET amino acid sequences from Populus and Arabidopsis SET proteins.

Additional file 2: Detailed locations of all duplicated pairs of SET genes in Populus from recent and ancient polyploidy events in the syntenic region. 
Additional file 3: Table-S1 $K_{s}$ of SET gene pairs in Suv, Ash, Atxr5, E (z) and Trx subfamilies. Table-S2 Raw data from microarray expression analyses for Populus SET genes. Sample abbreviations are defined in Figure 6 and Additional file 4. Table-S3 Raw data from ESTs for Arabidopsis SET genes. Table-S4 Raw data from transcriptome data for soybean SET genes.

Additional file 4: Transcript abundance in different subgroups across different tissues, organs, and treatments in Populus based on the genome-wide microarray data. Sample abbreviations are defined in Figure 6

Additional file 5: Structure of duplicated gene pairs within the Populus SET gene family. Exons, green filled boxes; introns, black single lines. UTRs are indicated by thick blue lines at both ends. Intron phases 0,1 and 2 are indicated by numbers 0,1 and 2 in the figure.

Additional file 6: Examples of five scenarios for terminus diversity in duplicated gene pairs of the SET family in Populus. This number corresponds to the one in Figure 6, and the red box represents the location of the mutations causing the patterns.

\section{Abbreviations}

K: Lysine; ML: Maximum likelihood; Bl: Bayesian inference; NJ: NeighbourJoining; Mya: Million years ago; WGD: Whole-genome duplication; PGDD: Plant genome duplication database; TF: Transcriptional factor; EST: Expressed sequence tag; NCBI: National center for biotechnology Information; UTR: Untranslated region; $K_{s}$ : Synonymous substitutions rate: $K_{a}$ : Non-synonymous substitution rate.

\section{Acknowledgements}

We would like to thank Drs. Fei He, Jinyong Hu, Zhenguo Lin and Zhenguo Zhang for comments on the manuscript and helpful discussions. This work was supported by the National Natural Science Foundation of China (91131007).

\section{Author details}

'State Key Laboratory of Systematic and Evolutionary Botany, Institute of Botany, the Chinese Academy of Sciences, Beijing 100093, China. ${ }^{2}$ State Key Laboratory of Genetic Engineering, Institute of Plants Biology, Center for Evolutionary Biology, School of Life Sciences, Fudan University, Shanghai 200433, China. ${ }^{3}$ Graduate University of the Chinese Academy of Sciences, Beijing 100039, China.

\section{Authors' contributions}

LSZ and LL conceived the study and performed the analyses. LL and LSZ wrote the manuscript. HM provided support for the study, contributed to the discussion and revised the manuscript. SLZ revised the manuscript and contributed to discussion. All authors read and approved the final manuscript.

Received: 12 September 2011 Accepted: 12 April 2012

Published: 12 April 2012

\section{References}

1. Bannister AJ, Kouzarides T: Regulation of chromatin by histone modifications. Cell Res 2011, 21:381-395.

2. Dillon SC, Zhang X, Trievel RC, Cheng X: The SET-domain protein superfamily: protein lysine methyltransferases. Genome Biol 2005, 6:227.

3. Feng $Q$, Wang $H$, Ng HH, Erdjument-Bromage $H$, Tempst $P$, Struhl K, Zhang Y: Methylation of H3-lysine 79 is mediated by a new family of HMTases without a SET domain. Curr Biol 2002, 12:1052-1058.

4. Muitaba S, Manzur KL, Gurnon JR, Kang M, Van Etten JL, Zhou MM: Epigenetic transcriptional repression of cellular genes by a viral SET protein. Nat Cell Biol 2008, 10:1114-1122.

5. Alvarez-Venegas R, Sadder M, Tikhonov A, Avramova Z: Origin of the bacterial SET domain genes: vertical or horizontal? Mol Biol Evol 2007, 24:482-497.

6. Liu C, Lu F, Cui X, Cao X: Histone methylation in higher plants. Annu Rev Plant Biol 2010, 61:395-420.
7. Goodrich J, Puangsomlee P, Martin M, Long D, Meyerowitz EM, Coupland G: A Polycomb-group gene regulates homeotic gene expression in Arabidopsis. Nature 1997, 386:44-51.

8. Alvarez-Venegas R, Pien S, Sadder M, Witmer X, Grossniklaus U, Avramova Z: ATX-1, an Arabidopsis homolog of trithorax, activates flower homeotic genes. Curr Biol 2003, 13:627-637.

9. Tamada Y, Yun JY, Woo SC, Amasino RM: ARABIDOPSIS TRITHORAXRELATED7 is required for methylation of lysine 4 of histone $\mathrm{H} 3$ and for transcriptional activation of FLOWERING LOCUS C. Plant Cell 2009, 21:3257-3269.

10. Zhao Z, Yu Y, Meyer D, Wu C, Shen WH: Prevention of early flowering by expression of FLOWERING LOCUS C requires methylation of histone H3 K36. Nat Cell Biol 2005, 7:1256-1260.

11. Grossniklaus U, Vielle-Calzada JP, Hoeppner MA, Gagliano WB: Maternal control of embryogenesis by MEDEA, a polycomb group gene in Arabidopsis. Science 1998, 280:446-450.

12. Guo L, YU Y, Law JA, Zhang X: SET DOMAIN GROUP2 is the major histone $\mathrm{H} 3$ lysine 4 trimethyltransferase in Arabidopsis. Proc Natl Acad Sci USA 2010, 107:18557-18562.

13. Berr A, McCallum EJ, Menard R, Meyer D, Fuchs J, Dong A, Shen WH: Arabidopsis SET DOMAIN GROUP2 is required for H3K4 trimethylation and is crucial for both sporophyte and gametophyte development. Plant Cell 2010, 22:3232-3248.

14. Aquea F, Vega A, Timmermann T, Poupin MJ, Arce-Johnson P: Genomewide analysis of the SET DOMAIN GROUP family in grapevine. Plant Cell Rep 2011, 30:1087-1097.

15. Zhang $L S$, Ma CR, Ji Q, Wang YF: Genome-wide identification, classification and expression analyses of SET domain gene family in Arabidopsis and rice. Yi Chuan 2009, 31:186-198.

16. Ng DW, Wang T, Chandrasekharan MB, Aramayo R, Kertbundit S, Hall TC: Plant SET domain-containing proteins: structure, function and regulation. Biochim Biophys Acta 2007, 1769:316-329.

17. Baumbusch LO, Thorstensen T, Krauss V, Fischer A, Naumann K, Assalkhou R, Schulz I, Reuter G, Aalen RB: The Arabidopsis thaliana genome contains at least 29 active genes encoding SET domain proteins that can be assigned to four evolutionarily conserved classes. Nucleic Acids Res 2001, 29:4319-4333.

18. Springer NM, Napoli CA, Selinger DA, Pandey R, Cone KC, Chandler VL, Kaeppler HF, Kaeppler SM: Comparative analysis of SET domain proteins in maize and Arabidopsis reveals multiple duplications preceding the divergence of monocots and dicots. Plant Physiol 2003, 132:907-925.

19. Naumann K, Fischer A, Hofmann I, Krauss V, Phalke S, Irmler K, Hause G, Aurich AC, Dorn R, Jenuwein T, Reuter G: Pivotal role of AtSUVH2 in heterochromatic histone methylation and gene silencing in Arabidopsis. EMBO J 2005, 24:1418-1429.

20. Jansson S, Douglas CJ: Populus: a model system for plant biology. Annu Rev Plant Biol 2007, 58:435-458.

21. Brunner AM, Busov VB, Strauss SH: Poplar genome sequence: functional genomics in an ecologically dominant plant species. Trends Plant Sci 2004, 9:49-56

22. Tuskan GA, Difazio S, Jansson S, Bohlmann J, Grigoriev I, Hellsten U, Putnam N, Ralph S, Rombauts S, Salamov A, et al: The genome of black cottonwood, Populus trichocarpa (Torr. \& Gray). Science 2006, 313:1596-1604.

23. Tang H, Wang X, Bowers JE, Ming R, Alam M, Paterson AH: Unraveling ancient hexaploidy through multiply-aligned angiosperm gene maps. Genome Res 2008, 18:1944-1954.

24. Feng S, Jacobsen SE: Epigenetic modifications in plants: an evolutionary perspective. Curr Opin Plant Biol 2011, 14:179-186.

25. Jiao Y, Wickett NJ, Ayyampalayam S, Chanderbali AS, Landherr L, Ralph PE, Tomsho LP, Hu Y, Liang H, Soltis PS, et al: Ancestral polyploidy in seed plants and angiosperms. Nature 2011, 473:97-100.

26. Tang H, Bowers JE, Wang X, Ming R, Alam M, Paterson AH: Synteny and collinearity in plant genomes. Science 2008, 320:486-488.

27. Chothia C, Gough J, Vogel C, Teichmann SA: Evolution of the protein repertoire. Science 2003, 300:1701-1703.

28. Bashton $M$, Chothia $C$ : The generation of new protein functions by the combination of domains. Structure 2007, 15:85-99.

29. Zhu X, Ma H, Chen Z: Phylogenetics and evolution of Su(var)3-9 SET genes in land plants: rapid diversification in structure and function. BMC Evol Biol 2011, 11:63. 
30. Babushok DV, Ostertag EM, Kazazian HH Jr: Current topics in genome evolution: molecular mechanisms of new gene formation. Cell Mol Life Sci 2007, 64:542-554.

31. Peisajovich SG, Garbarino JE, Wei P, Lim WA: Rapid diversification of cell signaling phenotypes by modular domain recombination. Science 2010, 328:368-372.

32. Pontvianne F, Blevins T, Pikaard CS: Arabidopsis histone lysine methyltransferases. Adv Bot Res 2010, 53:1-22.

33. Wilkins O, Nahal H, Foong J, Provart NJ, Campbell MM: Expansion and diversification of the Populus R2R3-MYB family of transcription factors. Plant Physiol 2009, 149:981-993.

34. Xu G, Ma H, Nei M, Kong H: Evolution of F-box genes in plants: different modes of sequence divergence and their relationships with functional diversification. Proc Natl Acad Sci USA 2009, 106:835-840.

35. Xu G, Guo C, Shan H, Kong H: Divergence of duplicate genes in exonintron structure. Proc Natl Acad Sci USA 2012, 109:1187-1192.

36. Proost $S$, Pattyn P, Gerats T, Van de Peer Y: Journey through the past: 150 million years of plant genome evolution. Plant J 2011, 66:58-65.

37. Maere S, De Bodt S, Raes J, Casneuf T, Van Montagu M, Kuiper M, Van de Peer Y: Modeling gene and genome duplications in eukaryotes. Proc Natl Acad Sci USA 2005, 102:5454-5459.

38. Van de Peer $Y$, Maere $S$, Meyer A: The evolutionary significance of ancient genome duplications. Nat Rev Genet 2009, 10:725-732.

39. Schubert D, Primavesi L, Bishopp A, Roberts G, Doonan J, Jenuwein T, Goodrich J: Silencing by plant Polycomb-group genes requires dispersed trimethylation of histone $\mathrm{H} 3$ at lysine 27. EMBO J 2006, 25:4638-4649.

40. Dong G, Ma DP, Li J: The histone methyltransferase SDG8 regulates shoot branching in Arabidopsis. Biochem Biophys Res Commun 2008, 373:659-664.

41. Innan $\mathrm{H}$, Kondrashov F: The evolution of gene duplications: classifying and distinguishing between models. Nat Rev Genet 2010, 11:97-108.

42. Duarte JM, Cui L, Wall PK, Zhang Q, Zhang X, Leebens-Mack J, Ma H, Altman $\mathrm{N}$, dePamphilis CW: Expression pattern shifts following duplication indicative of subfunctionalization and neofunctionalization in regulatory genes of Arabidopsis. Mol Biol Evol 2006, 23:469-478.

43. Force A, Lynch M, Pickett FB, Amores A, Yan YL, Postlethwait J: Preservation of duplicate genes by complementary, degenerative mutations. Genetics 1999, 151:1531-1545.

44. Liu SL, Baute GJ, Adams KL: Organ and cell type-specific complementary expression patterns and regulatory neofunctionalization between duplicated genes in Arabidopsis thaliana. Genome Biol Evol 2011, 3:1419-1436.

45. Papp B, Pal C, Hurst LD: Dosage sensitivity and the evolution of gene families in yeast. Nature 2003, 424:194-197.

46. Qian W, Liao BY, Chang AY, Zhang J: Maintenance of duplicate genes and their functional redundancy by reduced expression. Trends Genet 2010, 26:425-430.

47. Finn RD, Tate J, Mistry J, Coggill PC, Sammut SJ, Hotz HR, Ceric G, Forslund K, Eddy SR, Sonnhammer EL, Bateman A: The Pfam protein families database. Nucleic Acids Res 2008, 36:D281-D288.

48. Finn RD, Mistry J, Schuster-Bockler B, Griffiths-Jones S, Hollich V, Lassmann T, Moxon S, Marshall M, Khanna A, Durbin R, et al: Pfam: clans, web tools and services. Nucleic Acids Res 2006, 34:D247-D251.

49. Eddy SR: Profile hidden Markov models. Bioinformatics 1998, 14:755-763.

50. Edgar RC: MUSCLE: a multiple sequence alignment method with reduced time and space complexity. BMC Bioinformatics 2004, 5:113.

51. Guo AY, Zhu QH, Chen X, Luo JC: GSDS: a gene structure display server. Yi Chuan 2007, 29:1023-1026

52. Suyama $M$, Torrents $D$, Bork P: PAL2NAL: robust conversion of protein sequence alignments into the corresponding codon alignments. Nucleic Acids Res 2006, 34:W609-W612.

53. Yang Z: PAML 4: phylogenetic analysis by maximum likelihood. Mol Biol Evol 2007, 24:1586-1591.

54. Lynch M, Conery JS: The evolutionary fate and consequences of duplicate genes. Science 2000, 290:1151-1155.

55. Tamura K, Dudley J, Nei M, Kumar S: MEGA4: Molecular Evolutionary Genetics Analysis (MEGA) software version 4.0. Mol Biol Evol 2007, 24:1596-1599.

56. Guindon S, Gascuel O: A simple, fast, and accurate algorithm to estimate large phylogenies by maximum likelihood. Syst Biol 2003, 52:696-704.
57. Ronquist F, Huelsenbeck JP: MrBayes 3: Bayesian phylogenetic inference under mixed models. Bioinformatics 2003, 19:1572-1574.

58. Saeed Al, Bhagabati NK, Braisted JC, Liang W, Sharov V, Howe EA, Li J, Thiagarajan M, White JA, Quackenbush J: TM4 microarray software suite. Methods Enzymol 2006, 411:134-193.

59. Severin AJ, Woody JL, Bolon $Y T$, Joseph B, Diers BW, Farmer AD Muehlbauer GJ, Nelson RT, Grant D, Specht JE, et al: RNA-Seq Atlas of Glycine max: a guide to the soybean transcriptome. BMC Plant Biol 2010, 10:160.

doi:10.1186/1471-2148-12-51

Cite this article as: Lei et al: Expansion and diversification of the SET domain gene family following whole-genome duplications in Populus trichocarpa. BMC Evolutionary Biology 2012 12:51.

\section{Submit your next manuscript to BioMed Central and take full advantage of:}

- Convenient online submission

- Thorough peer review

- No space constraints or color figure charges

- Immediate publication on acceptance

- Inclusion in PubMed, CAS, Scopus and Google Scholar

- Research which is freely available for redistribution

Submit your manuscript at www.biomedcentral.com/submit
C) Biomed Central 